\title{
A fast algorithm of image registration
}

\author{
Wei Han \\ Overall Plan Office,6th department \\ Systems Engineering Research Institute \\ Beijing,China \\ e-mail:2742069961@qq.com
}

\author{
Yue Wang \\ School of Computer Science \\ Northwestern Polytechnical University \\ Xi'an,China \\ e-mail:2742069961@qq.com
}

\begin{abstract}
Image registration is a basic problem in image processing, which is widely applied in the field of target tracking, stereo matching, scene recognition etc. For its diversity and complexity, registration algorithm still exists several problems in the speed and accuracy at present. Therefore, a fast sub-pixel algorithm of image registration based on block processing and iterative improvement is proposed, in which improves the registration speed greatly with accuracy on a good condition. The validity and superiority of the algorithm is proved by the running result based on actual experiment data.
\end{abstract}

Keywords-image registration; sub-pixel; image blocking; stitching

\section{INTRODUCTION}

With the rapid development of data acquisition and calculation performance, computer vision has become a research hotspot. As a basic problem in computer vision, image registration has been widely concerned as well. Image registration is the process of overlaying images of the same scene obtained from different times, viewpoints, or sensors. It has widely application requirements in the field of video monitoring, military reconnaissance and target tracking.

According to the data granularity of registration, image registration methods can be roughly divided into regionbased and feature-based. Region-based method, which determines the relationship of image via correlation metrics, has a large computation and poor robustness. Feature-based method, which measures the similarity of the images by the features, has a low computation and high stability. Therefore, feature-based method has been study more with emphasis on edge and corner feature. In the corner feature, Zhang et al ${ }^{[1]}$ achieve optimal match by optimizing the distance function between two feature point sets. Mikolajczyk et al [2] realize matching using descriptors of feature point. In the edge feature, Yang et al ${ }^{[3]}$ complete image registration through edge extraction and chain code correlation. Dai et al ${ }^{[4]}$ achieve regional similarity registration by boundary optimization. Comparatively speaking, corner-based method has more applications due to the characteristics of easy operation and good robustness. However, it still exists problems in inaccurate extraction and long time consuming, which limits practical application. Therefore, further improvemets need to be done to meet practical requirements.

\section{ALGORITHM ANALYSIS}

The familiar corner-based algorithms mainly consist of the following steps ${ }^{[5]}$, corner extraction, feature matching, transform model estimation, image resampling and stitching. Nevertheless, current registration method still exists several problems in speed and accuracy. Two main reasons can be explained as follows: on the one hand, pixel-based and sub-pixel-based methods are commonly used in the corner extraction. Pixel-based is fast but inaccurate, while sub-pixel-based is accurate but slow, so the speed and accuracy can't be effectively balance. On the other hand, in the process of model estimation, RANSAC algorithm, which performs better in reliability, stability and robustness, is frequently used to remove false feature point. However RANSAC algorithm has relatively strong dependence on the performance of corner extraction, thus too many corners or inaccurate extraction will directly influence the speed and precision of it.

To solve these problems, this paper proposes an improved fast sub-pixel algorithm of image registration. The algorithm combines high speed of pixel-level with high accuracy of sub-pixel and improves the RANSAC algorithm on purpose, which greatly increases the velocity of calculation with accuracy reserved. The main steps are as follows: firstly, image is separated and a certain number of points in each region are extracted by Harris. Coarse matching is achieved by computing RANSAC transform matrix with the corner sets. Calculating the matching degree so as to screen matching point. Then, aiming at the obtained sub-pixel point sets, accurate matching is achieved with RANSAC computed. Finally, using bilinear interpolation for image resampling and completing stitching. The algorithm flow is shown in Fig.1.

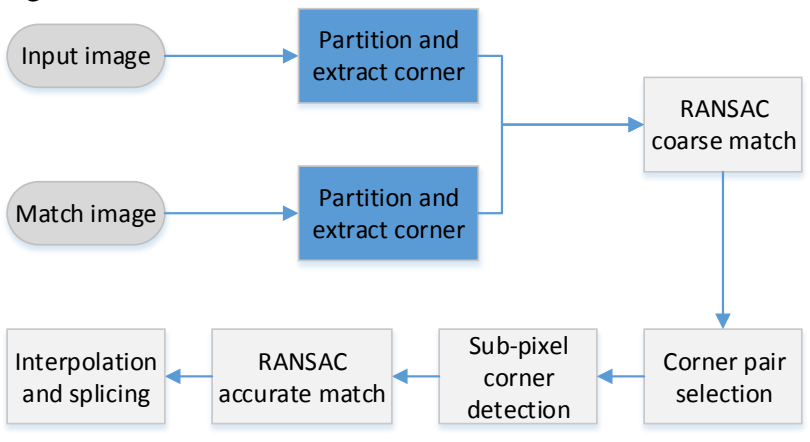

Figure 1. Algorithm flowchart of fast sub-pixel image registration

\section{ALGORITHM PROCESS}

At first, two images to be processed are divided into 6 12 regions, and then a certain number of points in each region are extracted by Harris with point limited and 
spread uniform. Harris and Sift are commonly used methods in corner detection. Harris is simple, fast but inaccurate, while Sift is precise but time-consuming. Here we only need roughly extraction, Harris is more desirable.

\section{A. Corner detection}

Harris corner ${ }^{[6]}$ is mainly obtained from curvature variation of image edge. By computing the variation of mean grey degree in different directions, the algorithm calculates the corner response function (CRF) and obtains concrete position via non-maximum suppression. The $2 \times 2$ symmetric matrix $M$ to which is refer in CRF calculation is given by

$$
M=e^{-\frac{x^{2}+y^{2}}{2 \sigma^{2}}} \otimes\left[\begin{array}{cc}
I_{x}{ }^{2} & I_{x} \cdot I_{y} \\
I_{x} \cdot I_{y} & I_{y}{ }^{2}
\end{array}\right]
$$

where $\otimes$ represents convolution, $I$ is the image, $I_{x}$ and $I_{y}$ are partial derivatives of $I$.

The point is regarded as a corner if both eigenvalues are high, and the CRF is defined as

$$
C R F(\mathrm{x}, \mathrm{y})=\operatorname{det}(\mathrm{M})-\mathrm{k}(\operatorname{trace}(\mathrm{M}))^{2}
$$

where $\operatorname{det}(\mathrm{M})$ represents determinant of $\mathrm{M}$, trace(M) represents trace of $\mathrm{M}, \mathrm{k}$ ranges from 0.04 to 0.06 . When the CRF value of the target pixel is greater than a given threshold, the pixel is a corner.

\section{B. Rough matching of image}

\section{1) Initial RANSAC matching}

Supposing the two image point sets which are obtained from Harris are $p=\left\{p_{1}, p_{2}, \mathrm{~L} p_{m}\right\}$ and $p^{\prime}=\left\{p_{1}^{\prime}, p_{2}{ }_{2}, \mathrm{~L}\right.$

$\left.p^{\prime}{ }_{m}\right\}$ respectively. Using the sum of squared differences (SSD) method to complete coarse matching, which is achieved by taking a square window of size of $\mathrm{w}$ around the pixel of interest in the reference image $I_{1}$ and finding the homologous pixel within the window in the target image $I_{2}$,

$$
S S D=\sum_{x, y \in w}\left[\mathrm{I}_{1}(\mathrm{x}, \mathrm{y})-\mathrm{I}_{2}(\mathrm{x}, \mathrm{y})\right]^{2}
$$

taking the minimum value as the matching point of $p_{i}$ in image $I_{1}$.

The point sets which is obtained from SSD still exist mismatch. Taking RANSAC to eliminate mismatch, and estimate transformation which can be replaced by a $3 \times 3$ homography matrix of $\mathrm{H}$ between the two images with the steps as follows:

a) Randomly select 4 groups of feature point pairs, estimate $\mathrm{H}$.

b) Take the remaining points into matrix $\mathrm{H}$ and calculate the error, the feature points pair is regarded as inlier when the error below the threshold which is settled, otherwise it will be outlier.

c) Repeat steps $a$ ) $\sim b$ ) for a prescribed number of iterations and preserve the outliners of maximum numbers.

d) Recalculate Matrix $\mathrm{H}$ with the obtained outliners.

\section{2) Corner pair selection}

Using neighborhood geometrical relationship of corner pair for calculating matching degree, and selecting the point of higher degree. Set $p_{i}$ and $p_{i}^{\prime}$ as a candidate matching point, then respectively select three points of $p_{i}$ and $p_{i}^{\prime}$ which has nearest Euclidean distance in image $I_{1}$ and $I_{2}$, as shown in Fig .2. Suppose $d_{1}<d_{2}<d_{3}$, the angle between $d_{1}$ and $d_{2}, d_{2}$ and $d_{3}$ is separately $\alpha$ and $\beta$. The relationship is as follows:

$$
\begin{aligned}
& \tan \alpha=\frac{k_{v_{2} p_{i}}-k_{v_{1} p_{i}}}{1+k_{v_{2} p_{i}} k_{v_{1} p_{i}}} \\
& d_{1}=\sqrt{\left(\mathrm{v}_{1} \mathrm{x}-p_{i} \mathrm{x}\right)^{2}+\left(\mathrm{v}_{1} y-p_{i} \mathrm{y}\right)^{2}}
\end{aligned}
$$

where $k_{v_{n} p_{i}}$ is the slope between point $v_{n}$ and $p_{i}(\mathrm{n}=1,2)$. If the matching point is correct, there should be a small gap in geometric relationship, such as $\left|d_{1}-d^{\prime}{ }_{1}\right|=\varepsilon$ and $\left|(\alpha-\beta)-\left(\alpha^{\prime}-\beta^{\prime}\right)\right|=\xi$, in which $\varepsilon$ and $\xi$ are very small positive. Choosing matching points lower than a settled threshold with at least three pairs ensured in each region.
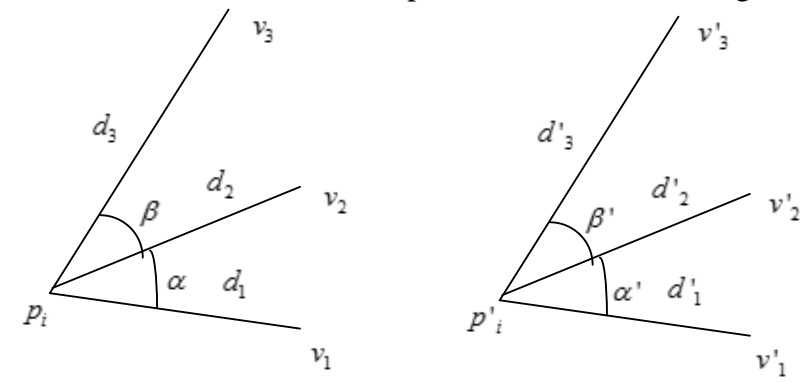

Figure 2. Geometrical relationship of candidate corner pair

\section{Accurate matching of image}

In order to improve matching degree, Zernike moments ${ }^{[7]}$ which have better effect are adopted for subpixel corner in this algorithm. Supposing the center of unite circle is at a pixel with image edge pass by that circle, and then an ideal step edge model is established shown in Fig .3 (a), in which $\mathrm{k}$ is the step height, $\mathrm{h}$ is the background gray level, 1 is the perpendicular distance from edge to the center and the edge makes an angle of $\phi$ with respect to $x$-axis. Rotating the image by an angle $\phi$, it will be aligned parallel to y-axis with shown in Fig .3 (b). The slide profile of the ideal step model after rotation is ladder-like, as is shown in Fig .3(c).

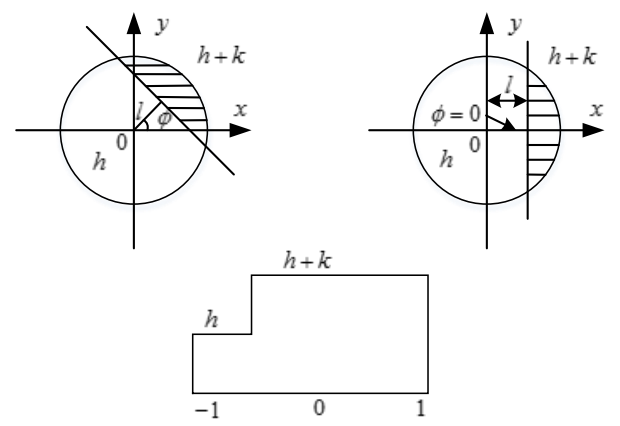

Figure 3. (a) Ideal step edge model (b) Ideal step edge model after rotation (c) Slide profile of the ideal step model after rotation 
If an image is rotated by an angle $\phi$, the Zernike moments merely acquire a phase shift on rotation and their amplitude remains constant. The Zernike moments of the rotated image are defined as

$$
\begin{aligned}
& A_{00}^{\prime}=A_{00}=h \pi+\frac{k}{2} \pi-k \sin ^{-1}(\mathrm{l})-\mathrm{kl} \sqrt{1-l^{2}} \\
& A_{11}^{\prime}=A_{11} e^{j \phi}=\frac{2 k}{3}\left(1-1^{2}\right)^{\frac{3}{2}} \\
& A_{20}^{\prime}=A_{20}=\frac{2 k}{3}\left(1-1^{2}\right)^{\frac{3}{2}}
\end{aligned}
$$

Referring to (5), we can further calculate

$$
l=\frac{A_{20}}{A_{11}^{\prime}}
$$

Then we can acquire the coordinate value of the sub$\operatorname{pixel}\left(\mathrm{x}_{s}, \mathrm{y}_{s}\right)$

$$
\left[\begin{array}{l}
x_{s} \\
y_{s}
\end{array}\right]=\left[\begin{array}{l}
x \\
y
\end{array}\right]+\frac{N l}{2}\left[\begin{array}{c}
\cos (\phi) \\
\sin (\phi)
\end{array}\right]
$$

With the obtained sub-pixel corner, images are divided once again and complete the precise image matching by RANSAC algorithm.

\section{Image resampling and stitching}

\section{1) Image resampling}

After the transformation is solved, image resampling is needed to be done which is the process of transforming a match image from one coordinate system to another. The inverse gridding approach is used to allocate a pixel's position in the original image, and the pixel value is calculated by interposition technology. Because the pixel is likely to not be integer after inverse gridding, bilinear interpolation is needed to calculate the value with the integer coordinates around the pixel. Supposing the four nearest points around the mapping pixel are $A(\mathrm{i}, \mathrm{j})$,

$\mathrm{B}(\mathrm{i}+1, \mathrm{j}), \mathrm{C}(\mathrm{i}, \mathrm{j}+1), \mathrm{D}(\mathrm{i}+1, \mathrm{j}+1)$, with the pixel values are $I(\mathrm{~A}), I(\mathrm{~B}), I(\mathrm{C}), I(\mathrm{D})$.

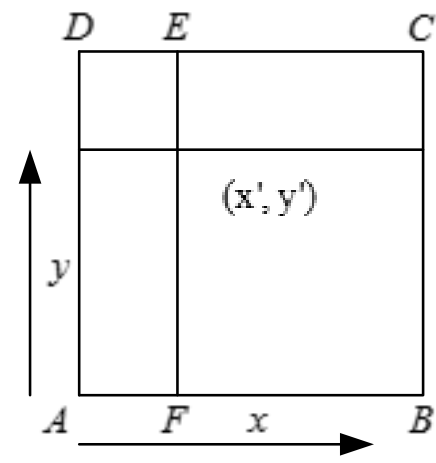

Figure 4. Principle chart of bilinear interpolation

Compute the value of $\mathrm{E}$ and $\mathrm{F}$,

$$
\begin{aligned}
& I(\mathrm{E})=\left(\mathrm{x}^{\prime}-\mathrm{i}\right)[I(\mathrm{~B})-I(\mathrm{~A})]+I(\mathrm{~A}) \\
& I(\mathrm{~F})=\left(\mathrm{x}^{\prime}-\mathrm{i}\right)[I(D)-I(\mathrm{C})]+I(\mathrm{C})
\end{aligned}
$$

then get $I\left(\mathrm{x}^{\prime}, \mathrm{y}^{\prime}\right)$,

$$
I\left(\mathrm{x}^{\prime}, \mathrm{y}^{\prime}\right)=\left(\mathrm{y}^{\prime}-j\right)[I(\mathrm{~F})-I(\mathrm{E})]+I(\mathrm{E})
$$

\section{2) Image stitching}

After completing image resampling, the reference image and origin image are in the same coordinate system, then if you take information from a single image or simply superimposing pixel values of the overlapping area, obvious gap will exists in the stitching region. In this paper, the weighted average method is taken to eliminate these joints. Each of the corresponding pixels in the two images is multiplied by a weight coefficient and then added to the fusion image. Let the weight coefficient be $\alpha$, and the fusion image can be written as

$$
I(\mathrm{x}, \mathrm{y})=\alpha I_{1}(\mathrm{x}, \mathrm{y})+(1-\alpha) I_{2}(\mathrm{x}, \mathrm{y})
$$

where $0 \leq \alpha \leq 1$.

\section{EXPERIMENTAL RESULTS AND ANALYSIS}

The image registration experimental results are shown in Fig .5 and Fig .6. Fig .5 are six frames of real monitoring image with a high resolution of $960 * 576$, each of them is a different view of the original scene.

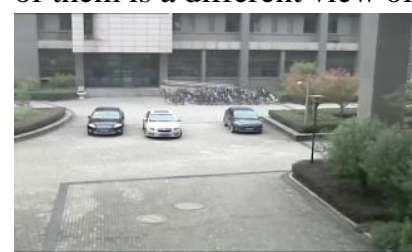

(a)

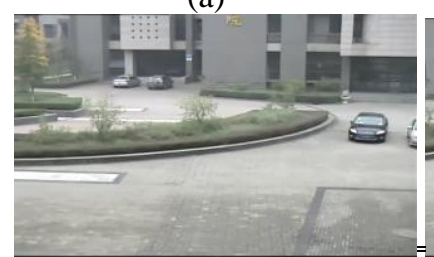

(b)

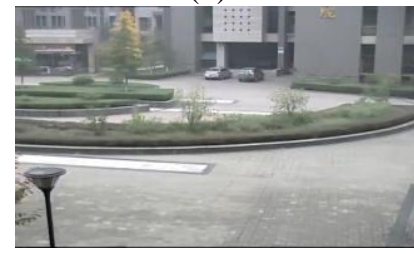

(e)

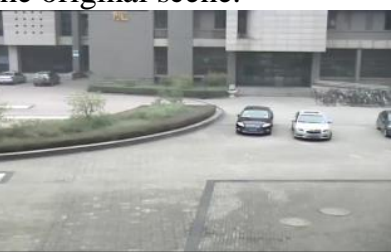

(b)

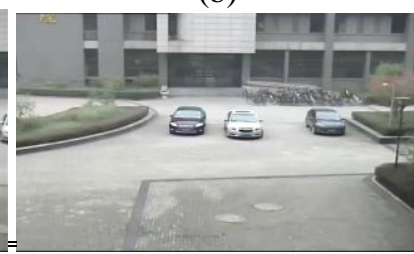

(d)

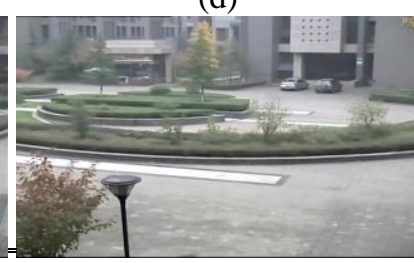

(f)
Figure 5. Real Monitoring Image(6 Frames)

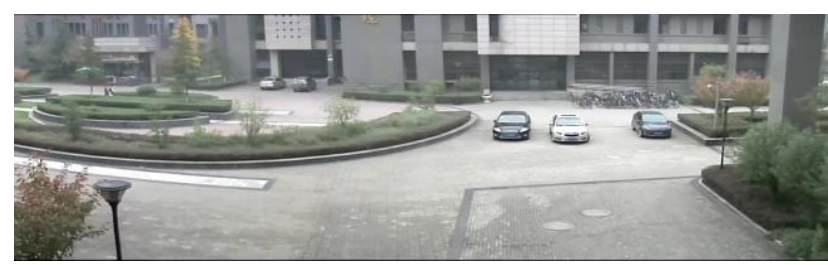

Figure 6. The effection image of splicing

The above experimental results show that the given algorithm is feasible and its performance is excellent. Except that, two groups of experiment are given for validating the algorithm performance.

\section{2) Corner detection test}

We compare the improved method which is proposed in this paper with the classical Harris method. The 
following results are shown in Table 1. As can be seen from the Table 1, Harris corner detection is time consuming in comparison because of the large feature numbers. However, most of the feature numbers have little use. Due to the decrease of corner numbers, the extraction time of paper's method is greatly reduced.

TABLE I. REAL CORNER NUM / TIME COMPARISON

\begin{tabular}{|c|c|c|c|c|c|c|c|}
\hline \multirow{2}{*}{ Info } & $\begin{array}{c}\text { Resul } \\
\text { t }\end{array}$ & $\begin{array}{c}\text { Fram } \\
\text { e 1 }\end{array}$ & $\begin{array}{c}\text { Fram } \\
\text { e 2 }\end{array}$ & $\begin{array}{c}\text { Fram } \\
\text { e 3 }\end{array}$ & $\begin{array}{c}\text { Fram } \\
\text { e 4 }\end{array}$ & $\begin{array}{c}\text { Fram } \\
\text { e5 }\end{array}$ & $\begin{array}{c}\text { Fram } \\
\text { e 6 }\end{array}$ \\
\hline \multirow{2}{*}{ Harries } & Points & 11732 & 10217 & 9231 & 12824 & 15638 & 16265 \\
\cline { 2 - 8 } & Time & $2.34 \mathrm{~s}$ & $2.14 \mathrm{~s}$ & $2.02 \mathrm{~s}$ & $2.54 \mathrm{~s}$ & $2.78 \mathrm{~s}$ & $2.84 \mathrm{~s}$ \\
\hline \multirow{2}{*}{$\begin{array}{c}\text { Our } \\
\text { Method }\end{array}$} & Points & 320 & 320 & 320 & 320 & 320 & 320 \\
\cline { 2 - 8 } & Time & $0.43 \mathrm{~s}$ & $0.42 \mathrm{~s}$ & $0.41 \mathrm{~s}$ & $0.46 \mathrm{~s}$ & 0.48 & 0.48 \\
\hline
\end{tabular}

3) Image registration test

Experiments are carried out by comparison with the method of Harris. It can be seen from Table 2, the registration time of the algorithm in this paper is greatly improved $77 \%$ and the accuracy of measurement can reach sub-pixel level. Therefore, the improved proposed in this paper has been greatly improved the efficiency of the algorithm.

TABLE II. IMAGE REGISTER SPEED/ACCURACY COMPARISON

\begin{tabular}{|c|c|c|}
\hline Info & Time(s) & Accuracy(Pixel) \\
\hline Harries & 25.72 & 1 \\
\hline Our Method & 5.84 & 0.52 \\
\hline
\end{tabular}

\section{CONCLUSION}

In view of the deficiency of traditional registration algorithm in speed and accuracy, this paper puts forward a fast sub-pixel algorithm of image registration with image blocking and sub-pixel corner detection technology which are aiming at the improvement of these problems. The experimental results show that the proposed method can improve the speed of image registration with much less computational cost and it's effective and feasible.

\section{REFERENCES}

[1] H. Zhang, O. Hall-Holt, and A. Kaufman field. "In Computer Kaufman. Range image registration via Probability Field". Proceedings Computer Graphics International, pp.546-552, 2004.

[2] K. Mikolajczyk and C. Schmid. "A performance evaluation of local descriptors". IEEE Transactions on Pattern Analysis and Machine Intelligence, pp. 1615-1630, 2005.

[3] Zhengwei Yang, Fernand S. Cohen. "Image registration and object improved chain-code representation combined with invarian recognition moments. using IEEE Trans". On Geoscience and Remote Sensing, vol 37, No 5, pp. 2351-2362, 1999

[4] Xianlong Dai, Siamak Khorram, "A feature-based image registration algorithm using improved chain-code representation combined with invariant moments IEEE Trans". On Geoscience and Remote Sensing, vol 37, No 5, pp. 2351-2362, 1999

[5] Litao Ma, Dan Yang, Xiaohong Zhang, Bo Li. "A new image registration based on condition number". Journal of Image and Graphics, vol 13, pp.277-283, February 2008.

[6] Chris Harris, Mike Stephens. "A Combined Corner And Edge Detection". Plessey Research Roke Manor, United Kingdom, pp.147-152, 1988

[7] Ghosal. S, Mehrotra. R, "Orthogonal moment operators for subpixel edge detection". Pattern Recogntion vol 26, pp.295-306, February 1993.

[8] Indranil Misra, S Manthira Moorthi, et al. "An automatic satellite image registration technique based on Harris corner detection and random sample consensus (RANSAC) outlier rejection model". 1st Int'l Conf. on Recent Advances in Information Technology, pp. 68-73, 2012.

[9] Zhiqiang Wei, Lei Huang, Xiaopeng Ji. "Research of sequence image matching method based point feature". Journal of Image and Graphics, vol 14, No 3, pp. 525-530, 2009

[10] Gongjian Wen, Jinjian Lv, Jiyang Wang. "Automatic registration method of high precision based on feature". Journal of Software, vol 19, No 9, pp. 2293-2300, September2009.

[11] Fischler Martin A,Bolles Robert C. "Random Sample Consensus: A Paradigm for Model Fitting with Application to Image Analysis and Automated Cartography".Communications of the ACM, vol 24, No 6, 1981 . 